Revista de Matemática: Teoría y Aplicaciones 2005 12(1 \& 2) : 61-72

CIMPA - UCR - CCSS ISSN: 1409-2433

\title{
ONE-SIDED CONFIDENCE INTERVAL ESTIMATION FOR WEIBULL SHAPE AND SCALE PARAMETERS
}

\author{
SMAIL MAHDI* \\ Received/Recibido: 1 Jul 2004
}

\begin{abstract}
In this paper, we consider the problem of one-sided conditional and unconditional interval estimation for the scale and shape parameters in a two-parameter Weibull model. The statistical inference is based upon the pivots advocated in Bain and Engelhardt, the likelihood ratio method and Birnbaum statistic. Simulation results illustrating the performance of these interval estimating methods are discussed and displayed. Empirical point estimate results obtained with the maximum likelihood, generalized moment and generalized probability weighted moment methods are also presented.
\end{abstract}

Keywords: Weibull distribution, rejection of a preliminary hypothesis, interval estimator, coverage probability, average length, simulation.

\section{Resumen}

En este artículo, consideramos el problema de estimación de intervalos unilaterales condicionales e incondicionales para los parámetros de escala y de forma en un modelo de Weibull de dos parámetros. La inferencia estadística está basada en los pivotes defendidos por Bain \& Engelhardt, el método del cociente de verosimilitud y el estadístico de Birnbaum. Se presentan y discuten resultados de simulación que ilustran el rendimiento de estos métodos de estimación de intervalos. También se presentan resultados de estimación puntual empírica obtenidos con métodos de máxima verosimilitud, momentos generalizados y de momentos ponderados de probabilidad generalizados.

Palabras clave: Distribución de Weibull, rechazo de una hipótesis preliminar, estimador de intervalo, probabilidad de cubrimiento, longitud promedio, simulación.

Mathematics Subject Classification: 62F25, 62F03.

\footnotetext{
${ }^{*}$ Department of Computer Science, Mathematics \& Physics, University of the West Indies, Cave Hill Campus, Barbados. E-Mail: smahdi@uwichill.edu.bb, mahdis89@hotmail.com.
} 


\section{Introduction}

The two-parameter Weibull probability density function is given by

$$
f(x ; \theta, \beta)=\beta \theta^{-\beta} x^{\beta-1} \exp \left[-\left(\frac{x}{\theta}\right)^{\beta}\right]
$$

for $x>0, \beta>0$ and $\theta>0$. The quantities $\beta$ and $\theta$ represent the shape and scale parameters, respectively. This distribution constitutes a successful analytical model for many phenomena in reliability engineering, infant mortality and extreme value problems. It is a very flexible distribution with a wide variety of possible curve shapes. In water research, for instance, the Weibull distribution is used to model the distribution of extreme values with small magnitude.

We consider here the problem of one-sided interval estimation for the two parameters $\beta$ and $\theta$. Conditional and unconditional type intervals are considered. The construction of these intervals rely on Bain and Engelhardt statistics $[1,2]$, the likelihood ratio statistic as well as Birnbaum statistic [3]. The conditional intervals are computed following D'Agostino and Meeks [11] setup and, therefore, are only considered in the case of rejection of preliminary tests for hypotheses about the relevant parameters. Earlier results on conditional interval estimations in Weibull, Exponential and Gaussian models are presented in Mahdi $[7,8,10]$. It is worth noting that the analysis of the performance of these interval estimators is often computationally tedious as already pointed out in Mahdi[9].

Before getting to the interval estimations, we first assess different point estimates for the scale and shape parameters. It is important to construct confidence bounds using optimal point estimates. We recall below two alternative estimating methods to the classical maximum likelihood methods, namely, the generalized method of moments and the generalized probability weighted moment method. The derivation of the following equations is detailed in Mahdi and Ashkar [6]. Simulation results for the comparison of the performance of these methods are given in appendix. We organize this paper as follows. In Section 2, following the introduction, we present two estimating methods and in Section 3 we derive unconditional confidence bounds for $\beta$ and $\theta$ using Bain and Engelhard method, likelihood ratio method and Birnbaum statistic. Conditional confidence intervals following these three methods are derived in Section 4. In Section 5, we derive the coverage probability of the conditional confidence interval and in Section 6 we discuss simulation results about the performance of the considered point and interval estimates. Tables illustrating the main simulation results are displayed in appendix.

\section{Point estimating methods}

We recall first the generalized method of moments and then the method of generalized probability weighted moments.

\subsection{Generalized method of moments}

The point estimates of the parameters $\beta$ and $\theta$, based on a random sample $x_{1}, \cdots, x_{n}$ that are provided by the generalized method of moments are given by the solutions $\beta_{m}$ and $\theta_{m}$ 
for the equations

$$
\frac{{\overline{x^{r_{1}}}}^{r_{2}}}{{\overline{x^{r_{2}}}}^{r_{1}}}=\frac{\left[\Gamma\left(1+\frac{r_{1}}{\beta_{m}}\right)\right]^{r_{2}}}{\left[\Gamma\left(1+\frac{r_{2}}{\beta_{m}}\right)\right]^{r_{1}}}
$$

and

$$
\theta_{m}=\left[\frac{\left[\Gamma\left(1+\frac{r_{1}}{\beta_{m}}\right)\right]^{r_{2}}}{\overline{x^{r_{1}}}}\right]^{\frac{1}{r_{1}}}
$$

The statistics $\overline{x^{r_{1}}}$ and $\overline{x^{r_{2}}}$ represent sample moments, about the origin, of order $r_{1}$ and $r_{2}$, respectively.

\section{$2.2 \quad$ Generalized probability weighted moments method}

The probability weighted moment method advocated in Hosking [5] constitutes a strong competitor of the maximum likelihood method. Using the probability weighted moment method, the parameters $\beta$ and $\theta$ are respectively estimated by

$$
\hat{\hat{\beta}}=\frac{\ln \left(k_{2}+1\right)-\ln \left(k_{1}+1\right)}{\alpha_{k_{1}}-\alpha_{k_{2}}-\ln \left(k_{2}+1\right)+\ln \left(k_{1}+1\right)}
$$

and

$$
\hat{\hat{\theta}}=\Gamma\left(1+\frac{1}{\hat{\hat{\beta}}}\right)\left(k_{1}+1\right)^{-1-\frac{1}{\hat{\hat{\beta}}}}
$$

where, $\hat{\alpha_{k}}=n^{-1} \sum_{i=1}^{n}\left(\begin{array}{c}n-i \\ k\end{array}\right) x_{(i)} /\left(\begin{array}{c}n-1 \\ k\end{array}\right)$ for $k=0, \cdots, n-1$, is the estimator of the probability weighted moment of real order $(1,0, k)$, see, for instance Hosking [5].

\section{Unconditional confidence bounds}

We derive in this section the bound of the unconditional confidence intervals for $\theta$ and $\beta$ using Bain and Engelhardt pivot, the likelihood ratio technique and Birnbaum statistic.

\subsection{Bain and Engelhardt pivots}

Using the statistical pivots derived in Bain and Engelhardt [1, 2], that is,

$$
\frac{c n \beta^{2}}{\hat{\beta}^{2}} \sim \chi_{c(n-1)}^{2}
$$

where $c=0.822$, respectively, $c=1$, and

$$
\sqrt{n-1} \frac{\hat{\beta} \ln \frac{\hat{\theta}}{\theta}}{c^{\prime}} \sim t_{n-1}
$$


with $c^{\prime}=1.053$. The $100(1-p) \%$ unconditional confidence upper bounds $\beta_{U}$ for $\beta$ and $\theta_{U}$ for $\theta$ are, respectively, given by

$$
\beta_{U}=\hat{\beta} \sqrt{\frac{\chi_{0.822(n-1)}^{2}(p)}{0.822 n}}
$$

and

$$
\theta_{U}=\hat{\theta} \exp \left[\frac{1.053 t_{n-1}(p)}{\hat{\beta} \sqrt{n-1}}\right] .
$$

The quantities $t_{n-1}(p)$ and $\chi_{c(n-1)}^{2}(p)$ denote the quantiles of order $(1-p) 100 \%$ of the Student variable with $n-1$ degrees of freedom and the Chi-square distribution with $c(n-1)$ degrees of freedom, respectively. The above pivots involve the maximum likelihood estimates of $\beta$ and $\theta$ which are, respectively, obtained from the equations

$$
\tilde{\beta}=\left[\left(\sum_{i=1}^{n} x_{i}^{\tilde{\beta}} \ln \left(x_{i}\right)\right)\left(\sum_{i=1}^{n} x_{i}^{\tilde{\beta}}\right)^{-1}-\frac{1}{n} \sum_{i=1}^{n} \ln \left(x_{i}\right)\right]^{-1}
$$

and

$$
\hat{\theta}=\left[n^{-1} \sum_{i=1}^{n} x_{i}^{\hat{\beta}}\right]^{\frac{1}{\hat{\beta}}}
$$

\subsection{Likelihood ratio statistic}

We first derive the likelihood ratio confidence bound for the shape parameter. The likelihood function based on the two parameter Weibull distribution and the random sample $x_{1}, \cdots, x_{n}$ is given by

$$
L w(\theta, \beta)=\beta^{n} \theta^{-n \beta} \prod_{i=1}^{n} x_{i}^{\beta-1} \exp -\left[\sum_{i=1}^{n}\left(\frac{x_{i}}{\theta}\right)^{\beta}\right] .
$$

For a fixed value $\beta$, the maximum likelihood estimator of $\theta$ is easily obtained as

$$
\tilde{\theta}=\left[\frac{\sum_{i=1}^{n} x_{i}^{\beta}}{n}\right]^{\frac{1}{\beta}} .
$$

Thus the profile likelihood for $\beta$ is given by

$$
L w(\tilde{\theta}, \beta)=\beta^{n}\left[\frac{\sum_{i=1}^{n} x_{i}^{\beta}}{n}\right]^{-n} \prod_{i=1}^{n} x_{i}^{\beta-1} \exp [-n],
$$

and the corresponding profile likelihood ratio by

$$
\operatorname{PLR}(\beta)=\frac{\operatorname{Lw}(\tilde{\theta}, \beta)}{\operatorname{Lw}(\hat{\theta}, \hat{\beta})},
$$


where $(\hat{\theta}, \hat{\beta})$ denote the maximum likelihood estimate of $(\theta, \beta)$. The upper bound of the $(1-\alpha) 100 \%$ confidence interval for $\beta$ is then given by the largest value of the solution set of the inequation

$$
\operatorname{PLR}(\beta)>\exp -\left[\frac{\chi_{(2 p, 1)}^{2}}{2}\right]
$$

Similarly, we obtain the likelihood ratio upper confidence bound for $\theta$ as follows. For a fixed value $\theta$, we obtain the maximum likelihood estimator of $\beta$ as the solution $\tilde{\beta}$ of the profile likelihood gradient equation

$$
\frac{\partial \ln (L w(\theta, \beta))}{\partial \beta}=\beta\left[\sum_{i=1}^{n} \ln \left(\frac{x_{i}}{\theta}\right)\left[\left(\frac{x_{i}}{\theta}\right)^{\beta}-1\right]\right]-n=0 .
$$

Theorem 1 The profile likelihood gradient equation (17) admits a unique positive root $\tilde{\beta}$.

This result can be easily proven by applying the intermediate value property of continuous functions to the function

$$
g(\beta)=\sum_{i=1}^{n} \ln \left(y_{i}^{\beta}\right)\left[y_{i}^{\beta}-1\right]-n,
$$

where $y_{i}=\frac{x_{i}}{\theta}$ for $i=1, \cdots, n$, and whose derivative

$$
g^{\prime}(\beta)=\sum_{i=1}^{n} \ln \left(y_{i}\right)\left[y_{i}^{\beta}\left[\ln \left(y_{i}^{\beta}\right)+1\right]-1\right]
$$

is greater or equal to zero as sum of non negative quantities.

The profile likelihood ratio for $\theta$ is then given by

$$
P L R(\theta)=\frac{L w(\theta, \tilde{\beta})}{L w(\hat{\theta}, \hat{\beta})},
$$

where $(\hat{\theta}, \hat{\beta})$ denote the unrestricted maximum likelihood estimate of $(\theta, \beta)$. Consequently, the $(1-p) 100 \%$ likelihood ratio confidence interval upper bound for $\theta$ is given by the largest solution of the inequation

$$
\operatorname{PLR}(\theta)>\exp -\left[\frac{\chi_{(2 p, 1)}^{2}}{2}\right] .
$$

where $\chi_{(p, 1)}^{2}$ is the $(1-p) 100 \%$ percentile of the chi-squared distribution with one degree of freedom.

\subsection{Birnbaum statistic}

Birnbaum [3] proposed a method for testing hypotheses about the shape parameter of a two-parameter Weibull distribution using the two extreme statistics. Based on the ordered sample $x(1) \leq x(2) \leq \cdots \leq x(n)$ from a random sample $x_{1}, \cdots, x_{n}$ and the variables $y(i)=\left(\frac{x(i)}{\theta}\right)^{\beta}$ for $i=1, \cdots, n$, Birnbaum [3] defined the statistic $\Xi=\frac{X(1)}{X(n)}$ whose 
distribution is independent of the scale parameter $\theta$. This statistic is then used to perform hypotheses testings about the parameter $\beta$. We propose here to use the statistic $\Xi$ for constructing the one-sided confidence interval for $\beta$. The distribution function of $\Xi$ is easily found as

$$
\begin{aligned}
G_{\beta}(\xi) & =P(\Xi \leq \xi)=P\left(\frac{Y(1)}{Y(n)} \leq \xi^{\beta}\right) \\
& =n(n-1) \sum_{j=0}^{n-2}(-1)^{n-2-j}\left(\begin{array}{c}
n-2 \\
j
\end{array}\right) \frac{1}{n-1-j} \frac{\xi^{\beta}}{(j+1) \xi^{\beta}+n-1-j} .
\end{aligned}
$$

We have then $G_{\beta}(\xi)=H\left(\xi^{\beta}\right)$ where $H$ denotes the distribution function of $\frac{Y(1)}{Y(n)}$. From this expression, it is easy to see that $G_{\beta}(\xi)$ is a monotonically decreasing function of $\beta$. Indeed, $\frac{d G_{\beta}(\xi)}{d \beta}=H^{\prime}\left(\xi^{\beta}\right)(\ln \xi) \xi^{\beta}<0$. Therefore, the upper bound of the confidence interval is given by the solution $\beta$ of the equation

$$
G_{\beta}(\hat{\xi})=p
$$

where $\hat{\xi}$ is the observed value of $\Xi$. For full details on constructing confidence bounds using distribution function technique, see for instance, Casella and Berger [4] and Wardell $[12]$.

\section{Conditional confidence interval}

The bounds of the conditional intervals are derived according to the method set forth by Meeks and D'Agostino [11]. The set up is a follows. First we test the null hypotheses $H_{0}^{1}$ : $\theta=\theta_{0}$ and $H_{0}^{2}: \beta=\beta_{0}$ versus the alternative hypotheses $H_{a}^{1}: \theta>\theta_{0}$ and $H_{a}^{2}: \beta>\beta_{0}$ , where $\theta_{0}$ and $\beta_{0}$ are fixed values. Now in the case of rejection, we estimate $\theta$ and $\beta$ by confidence intervals. Thus the estimation by confidence interval is conditioned by the outcome of a preliminary test of significance.

\subsection{Bain and Engelhard statistic}

To test $H_{0}^{1}$ and $H_{0}^{2}$ and to construct the bounds of the conditional intervals we use the statistical pivots (6) and (7).

Using the distribution technique for constructing confidence intervals, we obtain the required $(1-p) 100 \%$ conditional confidence bound for $\theta$ as the solution $\theta_{C}$ of the inequation

$$
F_{C}\left(\frac{\sqrt{n-1} \hat{\beta} \ln \frac{\hat{\theta}}{\theta_{C}}}{c}\right)=\frac{F\left(\frac{\sqrt{n-1} \hat{\beta} \ln \frac{\hat{\theta}}{\theta_{C}}}{c}\right)-F\left(t_{n-1}(\alpha)+\frac{\sqrt{n-1} \hat{\beta} \ln (\psi)}{c}\right)}{1-F\left(t_{n-1}(\alpha)+\frac{\sqrt{n-1} \hat{\beta} \ln (\psi)}{c}\right)} \geq p
$$

where $F$ is the cumulative function of a Student variable with $n-1$ degrees of freedom and $\psi=\frac{\theta_{0}}{\theta_{C}}$. Similarly, the conditional confidence bound for $\beta$ is obtained from the conditional 
cumulative function of the pivot (6) given rejection of $H_{0}^{1}$. This conditional cumulative function is obtained as

$$
G_{C}(z)=\frac{G(z)-G\left(z_{1}\right)}{\left.1-G\left(z_{1}\right)\right)} \text { for } z \geq z_{1}
$$

where $G$ is a chi-square distribution with $c(n-1)$ degrees of freedom and $z_{1}=\frac{c n \beta_{0}^{2}}{\chi_{c(n-1)}^{2}(\alpha)}$. Thus the required conditional confidence bound $\beta_{C}$ is obtained from the equation $G_{C}\left(\frac{c n \beta_{C}^{2}}{\hat{\beta}}\right)=1-p$ since the left side of this equation increases as $\beta$ increases.

\subsection{Likelihood ratio conditional confidence interval}

\subsubsection{Case of shape parameter}

We derive in this section the conditional confidence intervals for $\beta$ and $\theta$ based on the likelihood ratio method. To this end, let consider the statistic pivot

$$
T=-2 \ln \frac{L w(\tilde{\theta}, \beta)}{L w(\hat{\theta}, \hat{\beta})} .
$$

When the population shape parameter assumes the value $\beta$, we reject the null hypothesis $H_{0}^{1}$ versus the alternative $H_{a}^{1}$ if $P L R(\beta)<\exp -\left[\frac{\chi_{(2 \alpha, 1)}^{2}}{2}\right]$. This inequality can be expressed as

$$
T>t_{0}=\chi_{(2 \alpha, 1)}^{2}-2 \ln \frac{L w(\tilde{\theta}, \beta)}{L w\left(\tilde{\theta}, \beta_{0}\right)}
$$

Thus the conditional distribution of $T$ given rejection of $H_{0}^{1}$ is

$$
F_{\beta}^{c}(t)=P\left(T \leq t \mid T>t_{0}\right)=\frac{F_{T}(t)-F_{T}\left(t_{0}\right)}{1-F_{T}\left(t_{0}\right)}
$$

where $t=2 \ln \frac{L w(\tilde{\theta}, \beta)}{L w(\hat{\theta}, \hat{\beta})} \geq t_{0}$ and $F_{T}$ is the distribution of a chi-squared variable with 1 degree of freedom. Note that $t=-2 \ln \frac{L w(\tilde{\theta}, \beta)}{L w(\hat{\theta}, \hat{\beta})}$ increases towards infinity as $\beta$ increases from $\hat{\beta}$. Thus $F_{\beta}^{c}(t)$ also increases monotonically towards 1 . Therefore the upper conditional confidence bound is obtained as solution $\beta$ of the inequation

$$
F_{\beta}^{c}\left(-2 \ln \frac{L w(\tilde{\theta}, \beta)}{L w(\hat{\theta}, \hat{\beta})}\right) \leq 1-p .
$$

Remark 1 In the case of always rejection of $H_{0}$, the equation (29) gives the confidence interval provided by the inequation (16).

Proof. In the case of always rejection of $H_{0}^{1}, L w\left(\hat{\theta}, \beta_{0}\right) \rightarrow 0$ and therefore the $F_{\beta}^{C} \rightarrow F_{T}$. Thus the confidence interval for $\beta$ is given by the solution set $\left\{\beta: F_{T}\left(-2 \ln \frac{L w(\tilde{\theta}, \beta)}{L w(\hat{\theta}, \hat{\beta})}\right)<1-p\right\}$ which has the same solution set as the inequation $\frac{L w(\tilde{\theta}, \beta)}{L w(\hat{\theta}, \hat{\beta})}>\exp -\left[\frac{\chi_{(2 p, 1)}^{2}}{2}\right]$. 


\subsubsection{Case of scale parameter}

The conditional confidence interval for $\theta$ is derived in similar way. The bound of the confidence interval is given by the upper bound of the solution set of the inequation

$$
F_{\theta}^{c}\left(-2 \ln \frac{L w(\theta, \tilde{\beta})}{L w(\hat{\theta}, \hat{\beta})}\right) \leq 1-p
$$

where

$$
F_{\theta}^{c}(t)=P\left(T \leq t \mid T>t_{0}\right)=\frac{F_{T}(t)-F_{T}\left(t_{0}\right)}{1-F_{T}\left(t_{0}\right)}
$$

and $t>t_{0}=\chi_{(2 \alpha, 1)}^{2}-2 \ln \frac{L w(\theta, \tilde{\beta})}{L w\left(\theta_{0}, \tilde{\beta}\right)}$.

\section{Coverage probability of the unconditional interval}

The bounds of the conditional confidence intervals are computed for the targeted $1-p$ coverage probability. However, the coverage probability of the unconditional confidence interval in the case of rejection must be computed under the conditional probability function of the used statistics given rejection of the null hypotheses. We illustrate this in the case where Bain and Engelhard pivots are used.

\subsection{Case of shape parameter}

In this case, the coverage probability of the one-sided unconditional confidence interval for $\beta$ is given by

$$
\begin{aligned}
C P & =P\left[\beta \leq \beta_{U} \mid \frac{c n \beta_{0}^{2}}{\hat{\beta}^{2}}<\chi_{c(n-1)}^{2}(1-\alpha)\right] \\
& =P\left[\frac{c n \beta^{2}}{\hat{\beta}^{2}} \leq \chi_{c(n-1)}^{2}(p) \mid \frac{c n \beta^{2}}{\hat{\beta}^{2}}<\frac{1}{\Psi} \chi_{c(n-1)}^{2}(1-\alpha)\right] \\
& =\frac{P\left[\frac{c n \beta^{2}}{\hat{\beta}^{2}} \leq \chi_{c(n-1)}^{2}(p), \frac{c n \beta^{2}}{\hat{\beta}^{2}}<\frac{1}{\Psi} \chi_{c(n-1)}^{2}(1-\alpha)\right]}{P\left[\frac{c n \beta^{2}}{\hat{\beta}^{2}}<\frac{1}{\Psi} \chi_{c(n-1)}^{2}(1-\alpha)\right]}
\end{aligned}
$$

where $\Psi=\frac{\beta_{o}^{2}}{\beta^{2}} \leq 1$. Using now the fact that the variable $\frac{c n \beta^{2}}{\hat{\beta}^{2}}$ has the chi-square cumulative function $G$, we deduce that

$$
\begin{aligned}
C P & =\frac{\min \left\{G \left(\chi_{c(n-1)}^{2}(p), G\left(\frac{1}{\Psi} \chi_{c(n-1)}^{2}(1-\alpha)\right\}\right.\right.}{G\left(\frac{1}{\Psi} \chi_{c(n-1)}^{2}(1-\alpha)\right.} \\
& =\frac{\min \left\{1-p, G\left(\frac{1}{\Psi} \chi_{c(n-1)}^{2}(1-\alpha)\right\}\right.}{G\left(\frac{1}{\Psi} \chi_{c(n-1)}^{2}(1-\alpha)\right.} \leq 1 .
\end{aligned}
$$

Note that in the case of always rejection, that is $\Psi=0$ or $\alpha=1$, the quantity $G\left(\frac{1}{\Psi} \chi_{c(n-1)}^{2}(1-\alpha)\right)=1$. Therefore, $C P=1-p$ as it should be. 


\subsection{Case of scale parameter}

In this case, the coverage probability of the unconditional interval for $\theta$ is given by

$$
\begin{aligned}
C P & =P\left[\theta \leq \theta_{U} \mid \sqrt{n-1} \frac{\hat{\beta} \ln \frac{\hat{\theta}}{\theta_{0}}}{c^{\prime}}>t_{n-1}(\alpha)\right] \\
& =P\left[\sqrt{n-1} \frac{\hat{\beta} \ln \frac{\hat{\theta}}{\theta}}{c^{\prime}} \geq-t_{n-1}(p) \mid \sqrt{n-1} \frac{\hat{\beta} \ln \frac{\hat{\theta}}{\theta}}{c^{\prime}}>t_{n-1}(\alpha)+\frac{\hat{\beta} \sqrt{n-1} \ln \psi}{c^{\prime}}\right] \\
= & \frac{P\left[\sqrt{n-1} \frac{\hat{\theta} \ln \frac{\theta}{c^{\prime}}}{c^{\prime}} \max \left\{-t_{n-1}(p), t_{n-1}(\alpha)+\frac{\hat{\beta} \sqrt{n-1} \ln \psi}{c^{\prime}}\right\}\right]}{\hat{\beta} \ln \frac{\hat{\theta}}{\theta}} t_{\left.t_{n-1}(\alpha)+\frac{\hat{\beta} \sqrt{n-1} \ln \psi}{c^{\prime}}\right]}^{P\left[\sqrt{n-1} \frac{\hat{c^{\prime}}}{c^{\prime}}\right.}
\end{aligned}
$$

Using the cumulative function $F$ of the Student variable $\sqrt{n-1} \frac{\hat{\beta} \ln \frac{\hat{\theta}}{c^{\prime}}}{\text {, we get }}$

$$
C P=\frac{\min \left\{1-p, 1-F\left(t_{n-1}(\alpha)+\frac{\hat{\beta} \sqrt{n-1} \ln \psi}{c^{\prime}}\right)\right\}}{1-F\left(t_{n-1}(\alpha)+\frac{\hat{\beta} \sqrt{n-1} \ln \psi}{c^{\prime}}\right)}
$$

As partial check of the above equation, we consider again the case of always rejection that is $\psi=0$ or $\alpha=1$. In such situation, we also get $C P=1-p$ as it should be since $t_{n-1}(\alpha)+\frac{\hat{\beta} \sqrt{n-1} \ln \psi}{c^{\prime}} \rightarrow-\infty$.

\section{Discussion}

To compare the performance of the different point and interval estimates of $\beta$ and $\theta$ a simulation has been performed. Several values for $n, \alpha, \psi, \Psi, \beta$ are considered. The taken nominal value is $p=0.05$ and $\theta=1$ is used. The point estimates of $\beta$ and $\theta$ obtained by the maximum likelihood method have been found to be more accurate than the ones obtained with the other estimating methods, see, Tables 1 and 2 for illustration. Root mean square errors are used to quantify the accuracy. On the other hand, another pilot simulation study has been performed for comparing the confidence intervals presented in this paper. The interval based on Birnbaum statistics has poorly performed; this may be due, to the non sufficiency of the statistic $\Xi$ for $\beta$. On the other hand, the likelihood ratio method provided high length intervals along with overestimated coverage probabilities. Therefore only Bain and Engelhard conditional and unconditional intervals are further considered. The accuracy of the distributional fit of Bain and Engelhardt pivots depends upon the accuracy of the point estimates of $\theta$ and $\beta$. These maximum likelihood estimates were sucessfully obtained using the Newton-Raphson procedure initiated with the starting point advocated in Zanakis [14]. The study showed that better accuracy is found for $c=0.822$ and for large sample sizes and non large $\beta$ values, see, for instance, 
Table 3 for illustration. Similar results are obtained for the scale parameter. The results agree with the expressions for the variances of the estimators $\hat{\beta}$ and $\hat{\theta}$ obtained from the computed inverse of Fisher information matrix $I$ whose entries are $I_{11}=n E\left[-\frac{\partial^{2} \ln f}{\partial \beta^{2}}\right]=$ $n \beta \theta^{2}, I_{12}=I_{21}=n E\left[-\frac{\partial^{2} \ln f}{\partial \beta \partial \theta}\right]=n K_{1} \theta$ and $I_{22}=n E\left[-\frac{\partial^{2} \ln f}{\partial \theta^{2}}\right]=\frac{n\left(1+K_{2}\right)}{\beta^{2}}$ where $K_{1}=.42784$ and $K_{2}=.823683$ are values of appropriate improper integrals evaluated with Mathematica [13]. Conditional and unconditional intervals based on Bain and Engelhardt pivots are compared to each other in terms of coverage probability and average length through simulations. These intervals are identical when the population scale and shape parameters are far enough from $\theta_{0}$ and $\beta_{0}$, respectively. For the shape parameter, which the main parameter, the study has showed that the conditional interval has a sighly larger length than the unconditional one when $\Psi$ increases from $\Psi=0.25$ towards $\Psi=1$. However, it has a significantly better coverage probability especyially when $\Psi \geq 0.75$. We recommend then the use of the conditional interval. In the case of scale parameter, we noticed that as $\theta$ get close to $\theta_{0}$, the unconditional interval become slightly wider than the conditional one especially for large pre-test significance levels $\alpha$. Furthermore, both intervals maintain a coverage probabality close to the nominal level. Threfore, we also recommend to use the conditional one if $\psi$ or its estimate is close 1 , say larger than, .90 as illustrated in Table 5 .

\section{A Tables}

\begin{tabular}{l|ccc}
\hline \hline $\mathrm{n}$ & ML & GM & GPWM \\
\hline & & & \\
25 & 0.9367 & 1.1054 & 1.0211 \\
50 & 0.6051 & 0.7650 & 0.7580 \\
100 & 0.4082 & 0.5600 & 0.5485 \\
200 & 0.2820 & 0.4267 & 0.3914 \\
\hline \hline
\end{tabular}

Table 1. RMSE for $\beta$ obtained with the maximum likelihood, generalized moment and generalized probability weighted moment methods in the case of $\beta=0.5(0.5) 10$ and $n=25,50,100$ and 200 .

\begin{tabular}{l|ccc}
\hline \hline $\mathrm{n}$ & ML & GM & GPWM \\
\hline & & & \\
25 & 0.0991 & 0.0990 & 0.1098 \\
50 & 0.0675 & 0.0720 & 0.0744 \\
100 & 0.0469 & 0.0537 & 0.0515 \\
200 & 0.0328 & 0.0409 & 0.0360 \\
\hline
\end{tabular}

Table 2. RMSE for $\theta$ obtained with the maximum likelihood, generalized moment and generalized probability weighted moment methods in the case of $\theta=1$ and

$$
n=25,50,100 \text { and } 200 \text {. }
$$


CONFIDENCE INTERVAL ESTIMATION FOR WEIBULL SHAPE AND SCALE PARAMETERS 71

\begin{tabular}{l|cccc}
\hline \hline $\mathrm{n}$ & 10 & 15 & $30-120$ & $10-120$ \\
\hline$\beta=0.5$ & .1942 & .1362 & .0581 & .1011 \\
$\beta=1.0$ & .3882 & 3.2725 & .1162 & .2021 \\
$\beta=1.5$ & .5830 & .4093 & .1743 & .3034 \\
$\beta=2.0$ & .7774 & .5451 & .2325 & .4043 \\
$\beta=2.5$ & .9710 & .6817 & .2906 & .5051 \\
$\beta=3.0$ & 1.1652 & .8169 & .3488 & .6062 \\
\hline \hline
\end{tabular}

Table 3. RMSE for estimates of $\beta$ in the cases of $\beta=0.5(0.5) 3$ and $n=10,15,30-120$ and $10-120$.

\begin{tabular}{c|ccc}
\hline \hline$\Psi$ & CPCI & CPUI & LR \\
\hline 0.05 & 0.9470 & 0.9470 & 1.0000 \\
0.15 & 0.9470 & 0.9470 & 1.0000 \\
0.25 & 0.9470 & 0.9470 & 0.9996 \\
0.35 & 0.9470 & 0.9470 & 0.9954 \\
0.45 & 0.9462 & 0.9462 & 0.9854 \\
0.55 & 0.9439 & 0.9423 & 0.9743 \\
0.65 & 0.9407 & 0.9316 & 0.9682 \\
0.75 & 0.9359 & 0.9085 & 0.9673 \\
0.85 & 0.9229 & 0.8586 & 0.9696 \\
0.95 & 0.8997 & 0.7617 & 0.9731 \\
1.05 & 0.8128 & 0.6015 & 0.9767 \\
\hline \hline
\end{tabular}

Table 4. Empirical coverage probabilities and length ratios of the conditional and unconditional intervals for $\beta$ obtained with Bain and Engelhardt method. $\Psi=\frac{\theta_{0}}{\theta}, C P I$, $C P U I$ represent the coverage probabilities of the conditional and unconditional intervals, respectively. The column $L R$ gives the length ratio of the unconditional interval to the conditional interval. Values are averaged over $\alpha$ and $n=10,15,30,60,120$.

\begin{tabular}{l|ccccccccccc}
\hline \hline$\psi$ & $\leq .90$ & .92 & .94 & .96 & .97 & 1.03 & 1.04 & 1.06 & 1.08 & 1.10 & $\geq 1.12$ \\
\hline LR & $\simeq 1$ & 1.022 & 1.045 & 1.065 & 1.142 & 1.253 & 1.218 & 1.12581 & 1.011 & 1.009 & $\simeq 1$ \\
\hline \hline
\end{tabular}

Table 5. Empirical length ratios of a $90 \%$ conditional confidence interval to the corresponding unconditional confidence interval expressed as function of $\psi=\frac{\theta_{0}}{\theta}$. Values are averaged over the sample sizes $n=10,15,30,60,120$ and $\alpha$ levels.

\section{References}

[1] Bain, L.J.; Engelhardt, M. (1981) "Simple approximate distributional results for confidence and tolerance limits for the Weibull distribution based on maximum likelihood estimators", Technometrics 23(1): 15-20. 
[2] Bain, L.J.; Engelhardt, M. (1991) Statistical Analysis of Reliability and Life-Testing Models. Deker, New York.

[3] Birnbaum, Z.W. (1974) "Computers and unconventional test-statistics", Reliability and Biometry, Statistical Analysis of Lifelength, SIAM.

[4] Casella, G.; Berger, R.L. (2002) Statistical Inference, second Edition. Duxbury.

[5] Hosking, J.R.M. (1986) "The theory of probability weighted moments", Research Report RC12210, IBM Thomas J. Watson Research Center, New York.

[6] Mahdi, S.; Ashkar, F. (2004) "Exploring generalized probability weighted moments, generalized moments and maximum likelihood estimating methods in two-parameter Weibull model", it Journal of Hydrology 285: 62-75.

[7] Mahdi, S. (2003) "Two-sample conditional inference in a Weibul model", Car. Jour. Math. Comp. Sci. 11: 1-12.

[8] Mahdi, S. (2000) "Estimation in exponential models", Matematicki Vesnik 52: 27-45.

[9] Mahdi, S. (1999) "Monte Carlo studies on the accuracy of an interval estimator after a preliminary test of significance procedure", Bulletin of the International Statistical Institute, 52nd session, Book 2, Helsinki: 253-254.

[10] Mahdi, S.; Gupta, V.P. (1993) "Conditionally specified confidence interval for the variance of a normal population", Bull. Soc. Math. Belg. B 45(3): 245-256.

[11] Meeks, S.L.; D'Agostino, R.B. (1983) "A note on the use of confidence limits following rejection of a null hypothesis", The American Statistician 37: 134-136.

[12] Wardell, D.G. (1997) "Small sample interval estimation of Bernoulli and Poisson parameters", The American Statistician 51(4): 321-325.

[13] Wolfram, S. (1991) Mathematica: A System for Doing Mathematics by Computer, (2nd ed.). Addison-Wesley Publishing Company, USA.

[14] Zanakis, S.H. (1979) "Extended pattern search with transformation for the threeparameter WEI distribution", Management Science 25: 1149-1161. 